\title{
The PI3K $\delta$ inhibitor idelalisib impairs the function of human dendritic cells
}

\author{
Christiane Braun $^{1} \cdot$ Sebastian Schlaweck $^{1,2}$ (D) $\cdot$ Solveig Nora Daecke ${ }^{1} \cdot$ Peter Brossart $^{1} \cdot$ Annkristin Heine $^{1}$
}

Received: 22 September 2020 / Accepted: 16 June 2021 / Published online: 25 June 2021

(c) The Author(s) 2021

\begin{abstract}
The PI3K $\delta$-inhibitor Idelalisib is approved for the treatment of Non-Hodgkin lymphoma. However, its use has been decreased within the last years due to deleterious infections such as cytomegalovirus and pneumocystis jirovecii. Here, we have investigated the effect of Idelalisib on human monocyte-derived dendritic cells (DCs) as important players in the induction of immune responses. We found that Idelalisib-treated DCs displayed impaired T cell stimulatory function. PI3K $\delta$ inhibition during differentiation resulted in decreased Interleukin-12, Interleukin-13 and TNF $\alpha$ production by DCs after lipopolysaccharide stimulation. Moreover, DCs showed decreased expression of the activation marker CD83 after Idelalisib treatment. Further, in line with this was the failure of Idelalisib-treated DCs to properly induce allogeneic $\mathrm{T}$ cells in a dose-dependent manner. Finally, activation of the NFkB pathway was also ablated in Idelalisib-treated DCs. Our results implicate that severe infectious complications may not only result from direct PI3K $\delta$-inhibition in $\mathrm{T}$ cells, but also from impaired DC function in Idelalisib-treated patients. Here, we provide new insight into the pathogenesis of Idelalisib-associated infectious complications. Our study may further provide a rationale for the use of Idelalisib as a novel therapeutic option in inflammatory diseases.
\end{abstract}

Keywords Idelalisib $\cdot$ Infection $\cdot$ Dendritic cells $\cdot$ PI3K $\delta$

\section{Introduction}

Non-Hodgkin lymphoma, like follicular lymphoma (FL) and small lymphocytic lymphoma/chronic lymphatic leukemia (SLL/CLL), are hematological malignancies characterized by uncontrolled proliferation of clonal B cells, which leads to painless lymphadenopathy in most cases. Any organ may be affected, which is reflected by the broad variety of clinical presentations of these diseases [1]. Treatment options for Non-Hodgkin lymphomas have evolved in the past decades

Christiane Braun and Sebastian Schlaweck have contributed equally

Annkristin Heine

Annkristin.Heine@ukbonn.de

1 Medical Clinic III, Clinic for Oncology, Hematology, Immuno-Oncology and Rheumatology/Clinical Immunology, University Hospital Bonn, Venusberg Campus 1, 53127 Bonn, Germany

2 Faculty of Medicine, Mildred Scheel School of Oncology Aachen Bonn Cologne Düsseldorf (MSSO ABCD), University Hospital of Bonn, 53127 Bonn, Germany from classical chemotherapy to immunotherapy as well as targeted therapy. Among targeted therapies, PI3K $\delta$ has been identified as a potent target for B cell malignancies. PI3K $\delta$ is a tyrosine kinase predominantly expressed in leukocytes. It is located in the cytoplasm and activates the AKT/mTOR pathway [2]. Idelalisib, a specific small molecule inhibiting PI3K $\delta$, showed remarkable clinical results for the treatment of FL as well as CLL [3, 4]. However, infection rates, for example of cytomegalovirus (CMV) and pneumocystis jirocevii (PJP) in patients treated with Idelalisib in combination with classical immune-chemotherapy, were significantly increased [5]. Impaired T cell-mediated immunity due to direct effects on $\mathrm{T}$ cell migration and cytokine production has already been described as a side effect of Idelalisib treatment [6].

$\mathrm{T}$ cell-mediated immunity and the induction of a potent immune response are not only $\mathrm{T}$ cell-dependent, but also require professional antigen-presenting cells (APCs). Dendritic cells (DCs) presenting a specific antigen and expressing appropriate co-stimulatory molecules are the most potent APCs. They can orchestrate immune responses of 
naïve $\mathrm{CD} 8+\mathrm{T}$ cells and subsequently induce cytotoxic $\mathrm{T}$ cell responses [7].

In this study, we investigated the effect of Idelalisib on DC function, which is a prerequisite for appropriate $\mathrm{T}$ cell activation, differentiation and proliferation. Our study may highlight how PI3K $\delta$ inhibition not only diminishes $\mathrm{T}$ cell function directly, but also affects antigen-specific $\mathrm{T}$ cell responses through attenuated DC function.

\section{Methods}

\section{Samples}

Human monocytes were isolated from buffy coats from voluntary blood donors at the University Hospital Bonn.

\section{Media and reagents}

Idelalisib was purchased from Selleckchem. Cells were cultured in RPMI 1640 containing glutamax-I, supplemented with $10 \%$ inactivated fetal calf serum (RP10 medium) and $1 \%$ penicillin/streptomycin (Invitrogen). Unless otherwise indicated, all reagents were purchased from Sigma-Aldrich.

\section{Generation of DCs}

Plastic adherence of peripheral blood allowed the generation of human moDCs, as previously described [8]. These adherent cells were differentiated in RP10 medium. GMCSF (100 ng/ml; Leukine, Liquid Sargramostim) and IL-4 (20 ng/ml; R\&D Systems) were supplemented from the beginning every other day.

\section{Immunostaining}

Fluorescence-labeled, monoclonal antibodies commercially available from BD Biosciences, DakoDiagnostika, Immunotech, $R \& D$ Systems and eBioscience were used for staining of generated DCs.

\section{Determination of cytokine production}

Cytokine secretion was analyzed using the eBioscience ${ }^{\mathrm{TM}}$ ProcartaPlex Human Th1/Th2 Zytokin-Panel (11-plex) (Thermofisher) according to the manufacturer's instructions.

\section{Mixed lymphocyte reactions}

$1 \times 10^{5}$ allogeneic peripheral blood mononuclear cells were co-cultured with pretreated and irradiated stimulator moDCs. On day 5 , after a $16 \mathrm{~h}$ pulse with $\left[{ }^{3} \mathrm{H}\right]$-thymidine
(18.5 kBq/well; GE Healthcare) Tritium-labeled thymidine incorporation was measured.

\section{Detection of apoptosis}

Apoptosis in DCs was detected by live-dead staining using the propidium iodide or 7-aminoactinomycin $\mathrm{D}$-annexin $\mathrm{V}$ staining kit from eBioscience.

\section{Polyacrylamide gel electrophoresis and western blotting}

Western blotting was performed as described before [9]. Briefly, whole cell lysates were generated, and protein concentration was measured using a bicinchoninic acid assay (Pierce, Perbio Science, Bonn, Germany). Whole cell lysates $(20 \mu \mathrm{g})$ were separated on a polyacrylamide gel and transferred onto a nitrocellulose membrane. Monoclonal antibodies by Santa Cruz Biotechnology Inc. (Santa Cruz, USA) were used. An enhanced chemiluminescence kit was used to detect protein bands (GE Healthcare).

\section{Statistical analysis}

All experiments were performed at least 3 times, with representative experiments shown. Statistical significance was calculated with one-way analysis of variance (ANOVA) and Dunnett's using the Prism 8.4.3 software (Graphpad Software).

\section{Results}

\section{Idelalisib modulates the differentiation of human monocytes into DCs and decreases LPS-induced maturation of moDCs}

To understand dose-dependent effects of the PI3K $\delta$ inhibitor on lineage and activation markers, we differentiated monocytes into moDCs in the presence of GM-CSF and IL-4. Idelalisib applications were performed from day 0 , on every other day, and final LPS maturation was induced on day 6 (Fig. 1A). Chosen concentrations of Idelalisib correlate with patient's sera concentrations.

As a first approach, we investigated the effect of Idelalisib on DC morphology and observed impaired formation of dendritic branches and trees. The generated cells resembled monocytes rather than DCs. (Fig. 1B). Next, we analyzed whether Idelalisib induces apoptosis in DCs, but did not detect any significant toxic effects on DCs in the concentrations used in vitro. (Fig. 1C). Expression of the monocyte marker CD14 was not affected by Idelalisib treatment and remained stable, whereas $\mathrm{CD} 1 \mathrm{a}$, a surface protein important 
Fig. 1 Idelalisib modulates the phenotype of moDCs without affecting cell viability A Schematic experimental design for the differentiation of moDCs. Human monocytes cultured under DC-driving conditions were exposed (i) every other day to different concentrations of Idelalisib $(0.5 \mu \mathrm{M}, 1 \mu \mathrm{M}$ and $2 \mu \mathrm{M}$ on day $0,2,4$ and 6) or DMSO or (ii) only on day 5 . When indicated, TLR stimulation was applied on day 6. Cells were analyzed on day 7. B Representative pictures of unstained and Phalloidin/DAPIstained moDCs after Idelalisib pre-treatment are shown, $\mathbf{C}$ Apoptosis rate calculated after $\mathrm{PI}$ and Annexin V staining is shown. MoDCs were generated in the presence of Idelalisib. Idelalisib or DMSO was added every other day. $(0.5 \mu \mathrm{M}, 1 \mu \mathrm{M})$
A
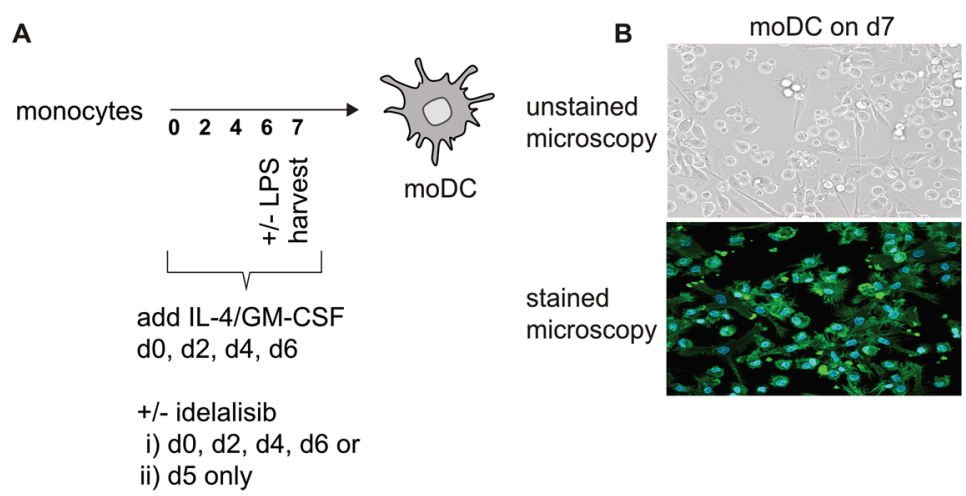

moDC + idelalisib on $\mathrm{d} 7$

C

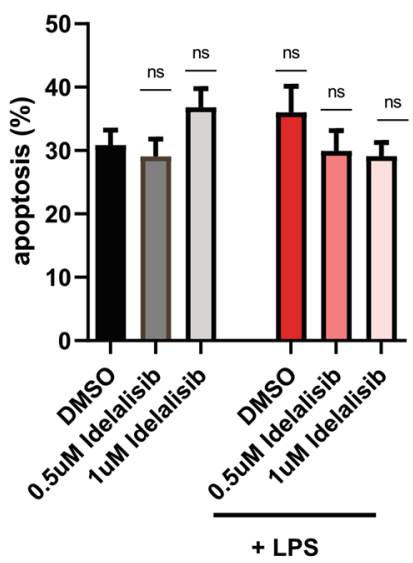

for the presentation of lipid and glycolipid antigens, was significantly upregulated by Idelalisib treatment after LPS maturation in a dose-dependent manner. LPS-matured DCs showed significantly reduced CD83 expression when compared to vehicle-exposed monocytes, while other activation markers such as CD86 and CD80, were not affected. PI3K $\delta$ blockade also resulted in downregulation of PD-L1 on immature moDCs, while CCR7 expression was not affected by Idelalisib. However, the effect on PD-L1 expression was not statistically significant after LPS maturation (Fig. 2A and B).

\section{Allogeneic $T$ cell activation by human moDCs is dampened by Idelalisib treatment}

We were further interested in how Idelalisib modulates the $\mathrm{DC}-\mathrm{T}$ cell interaction. First, we were able to show that $\mathrm{T}$ cells treated with Idelalisib showed reduced proliferation when co-incubated with allogeneic moDCs. (Fig. 3B) To assess the capacity of moDCs to orchestrate allogeneic $\mathrm{T}$ cell proliferation, we co-incubated Idelalisib-pretreated moDCs and untreated $\mathrm{T}$ cells. This mixed lymphocyte reaction (MLR) showed impaired induction of allogeneic T cell proliferation by Idelalisib-pretreated moDCs in vitro (Fig. 3C).
Thus, Idelalisib affects both T cells and DCs as it reduces their capacity to induce allogeneic $\mathrm{T}$ cell responses.

\section{Idelalisib alters the cytokine profile of LPS-matured DCs}

Besides co-stimulatory signaling and antigen presentation, secretion of cytokines influences the induction of an immune response. Therefore, pro-inflammatory cytokines were analyzed after Idelalisib treatment and LPS stimulation. IL-12 and IL- 2 are produced by DCs and are essential for T cell activation. Interferon $\gamma$ (INF $\gamma$ ), IL-4 and IL-13 induce or maintainT cell polarization. TNF $\alpha$ as a T cell effector molecule was measured. We detected significantly diminished levels of TNF $\alpha$, IL-13 and IL-12 in Idelalisib-pretreated moDCs while levels of IL-2 and INF $\gamma$ remained unchanged. In contrast, IL-4 secretion was even upregulated by Idelalisib treatment of immature moDCs, but this effect was abolished in mature moDCs (Fig. 4A).

\section{Idelalisib inhibits the NFkB pathway downstream of PI3Kס}

Due to significant downregulation of relevant cytokines for $\mathrm{T}$ cell proliferation and function, we were interested in the 
A

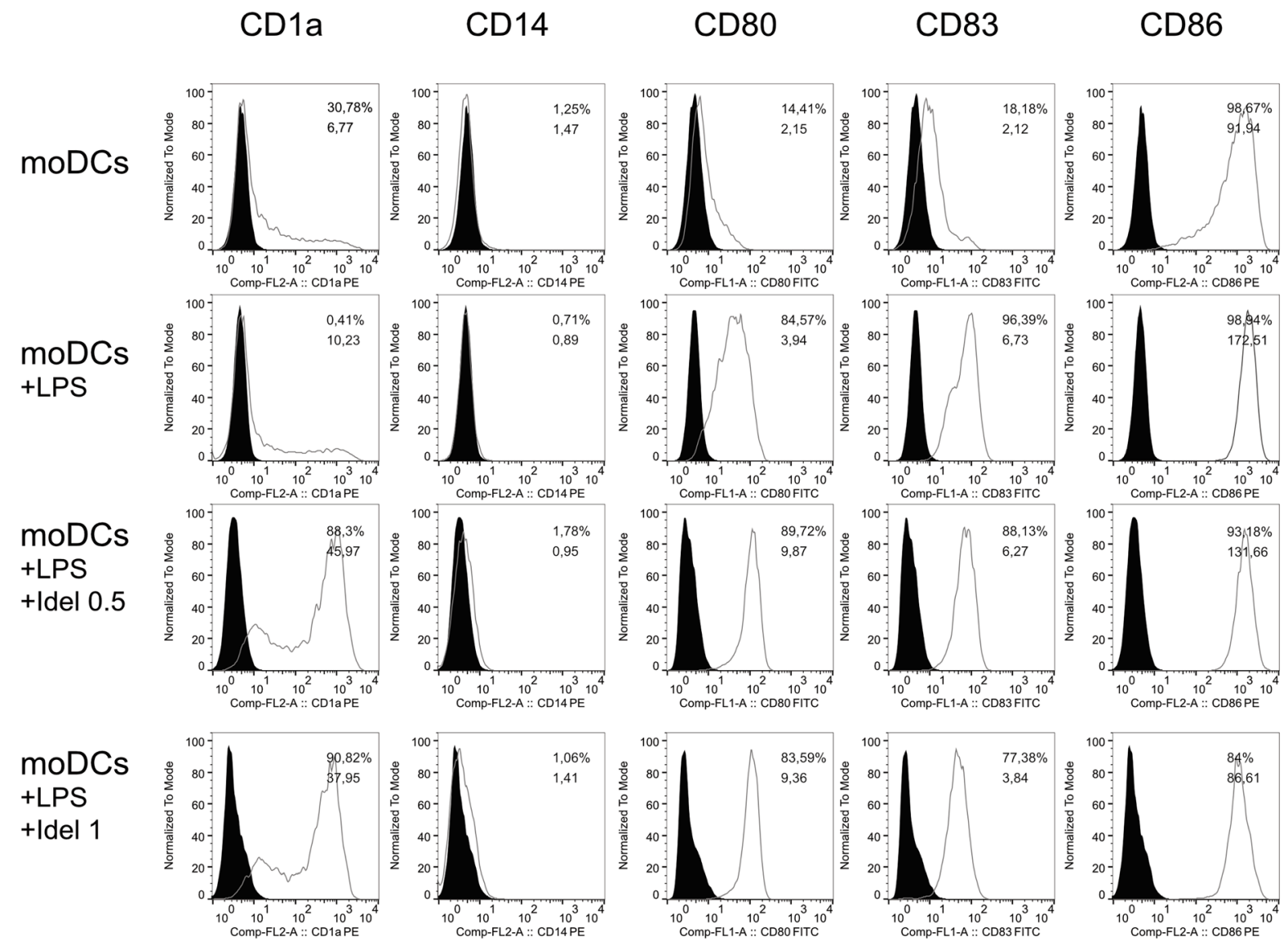

B
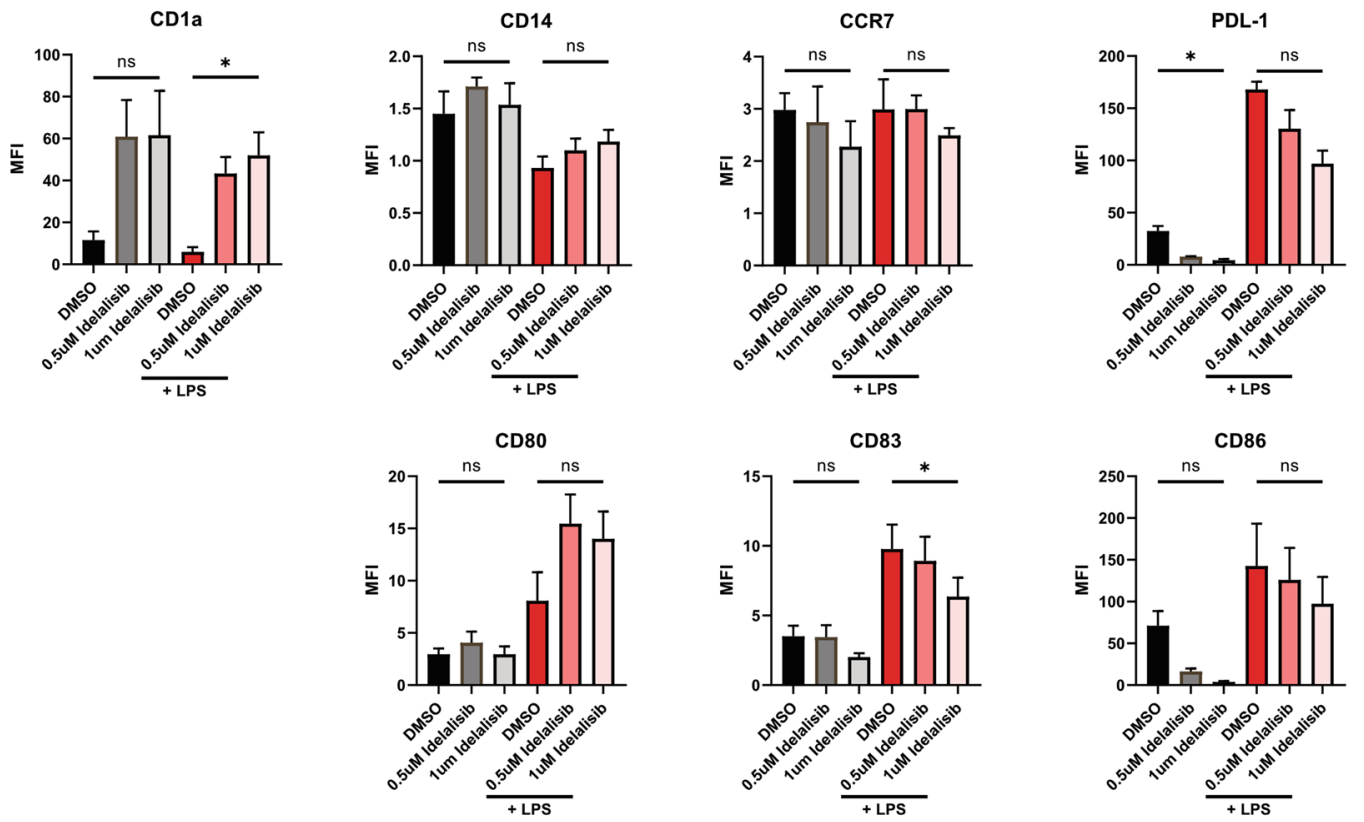
4Fig. 2 Modulation of co-stimulatory molecules by Idelalisib pretreatment A MoDCs were generated in the presence of IL-4 and GM-CSF. Idelalisib was added every other day followed by subsequent LPS maturation on day 6 , when indicated. Cells were harvested on day 7 and analyzed for expression of surface markers via FACS. Plots show mean fluorescent intensity (MFI) from one representative experiment. B MFI of surface molecules from three representative experiments were pooled and are shown. The significance was calculated according to the one-way ANOVA Dunnett multiple comparison test and is related to the vehicle control. $* P<0.05$

NFkB pathway, which is downstream of PI3K. For this purpose, we analyzed protein expression of Rel-B and c-Rel. LPS-induced upregulation of both proteins in moDCs, which was reduced by Idelalisib pre-treatment in a dose-dependent manner. Rel-A remained unchanged. (Fig. 4B). As expected, phosphorylation of PI3K was inhibited by Idelalisib. Neither endogenous PI3K expression nor expression of p-STAT3 were altered. (Fig. 4C).

\section{Discussion}

In the past decade, the treatment of Non-Hodgkin B cell lymphoma, such as FL and CLL, has evolved due to the discovery of novel drugs. Among small molecules and targeted therapies, Idelalisib, an inhibitor of $\mathrm{PI} 3 \mathrm{~K} \delta$, was a promising new candidate.

Nevertheless, pneumonitis, elevation of transaminases, colitis and diarrhea, as well as life-threatening infections, are serious side effects $[3,5]$ and have limited the treatment. Moreover, it has been shown that $\mathrm{T}$ cell-mediated immunity is dampened by Idelalisib treatment [6], and infectious

A

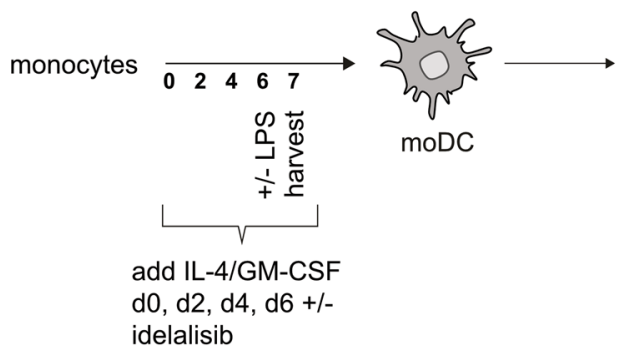

B

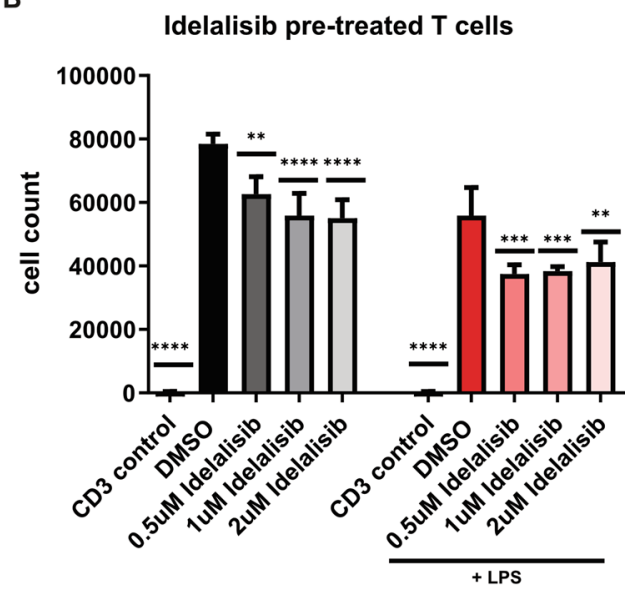

Fig. 3 Idelalisib affects proliferation of allogeneic $\mathrm{T}$ cells in a DCdependent manner A Schematic experimental design for a mixed lymphocyte reaction. Human monocytes cultured under DC-driving conditions with or without final LPS stimulation were exposed every other day to different concentrations of Idelalisib $(0.5 \mu \mathrm{M}, 1 \mu \mathrm{M}$ and $2 \mu \mathrm{M}$ on day $0,2,4$ and 6 ) or DMSO throughout the differentiation period. Afterward, moDCs were co-cultured with allogeneic cells for five days. Thymidine incorporation was measured, and cell numbers were calculated. B T cells were co-incubated for $24 \mathrm{~h}$ with Idelalisib. $(0.5 \mu \mathrm{M}, 1 \mu \mathrm{M}, 2 \mu \mathrm{M})$. Washed cells were co-incubated with differ-

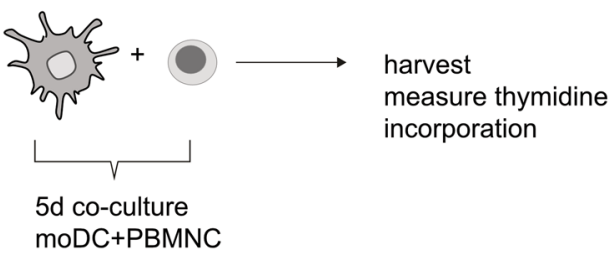

C

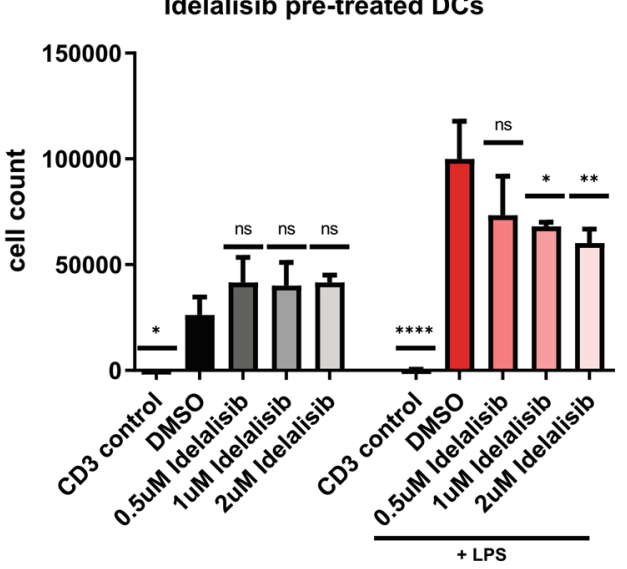

entiated moDCs naïve for Idelalisib treatment for 5 days and $\mathrm{T}$ cell counts $16 \mathrm{~h}$ after a $16 \mathrm{~h}$ pulse with $\left[{ }^{3} \mathrm{H}\right]$-thymidine are shown. Filled black graphs represent negative controls. $\mathbf{C}$ Monocytes were cultured under DC-driving conditions and treated with Idelalisib $(0.5 \mu \mathrm{M}$, $1 \mu \mathrm{M}, 2 \mu \mathrm{M}$ ) every second day (day $0,2,4,6$ ), followed by LPS activation on day 6 , when indicated. Pretreated moDCs were co-cultured with untreated $\mathrm{T}$ cells for an additional five days. T cell counts $16 \mathrm{~h}$ after a $16 \mathrm{~h}$ pulse with $\left[{ }^{3} \mathrm{H}\right]$-thymidineare shown. Filled black graphs represent negative controls 
A

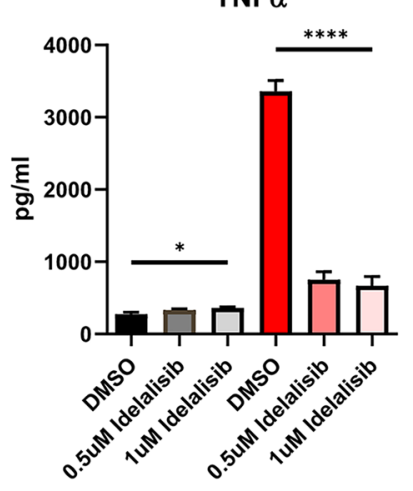

IL2

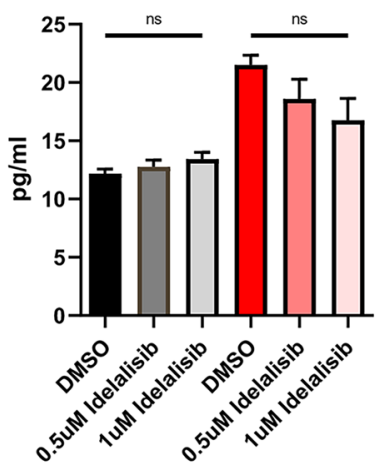

IL12

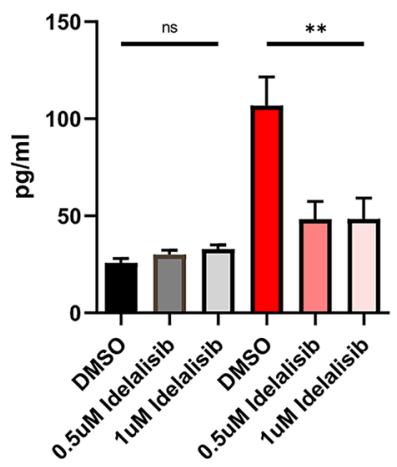

INF $\gamma$

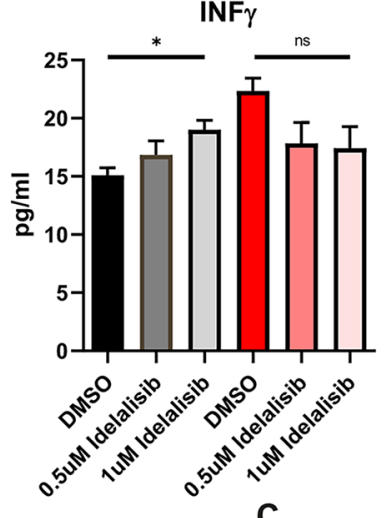

C
IL13

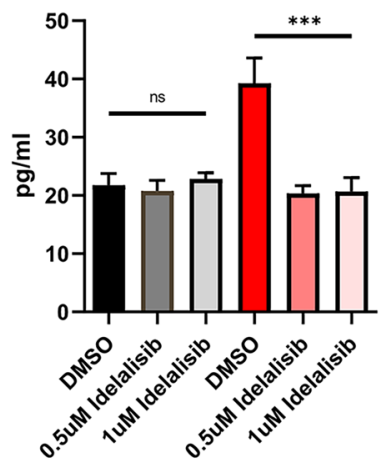

IL4

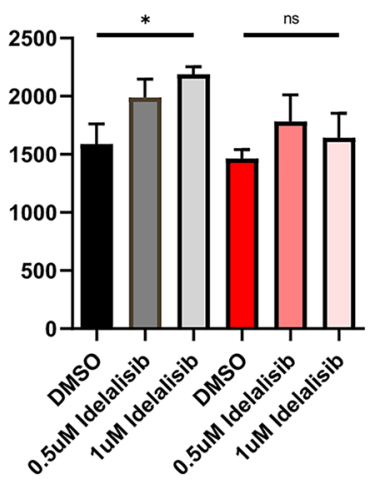

B

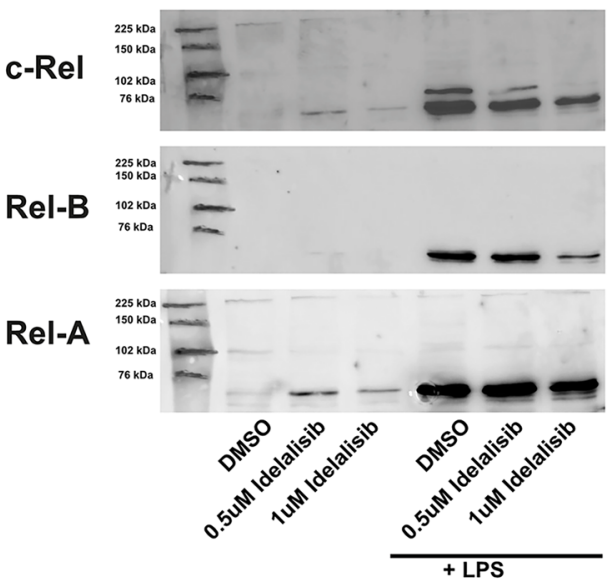

PI3K
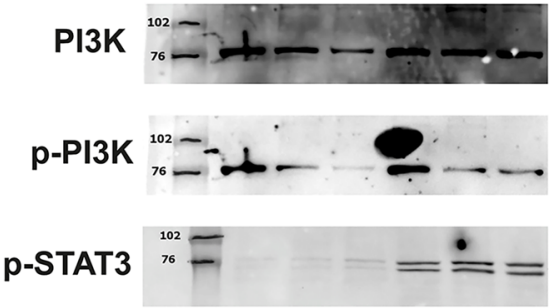

$\beta$-Actin
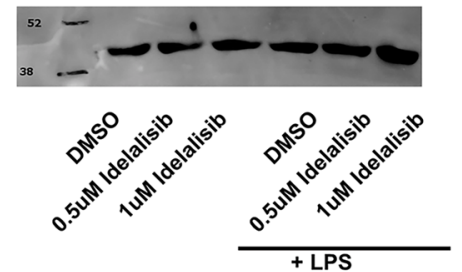

Fig. 4 Idealisib alters cytokine production by LPS stimulated moDCs and interferes with the $\mathrm{NF \kappa B}$ pathway. A MoDCs were generated as described in the presence of Idelalisib followed by subsequent LPS maturation on day 6 when indicated. Supernatants were collected on day 7 and analyzed for cytokine expression. The significance was calculated according to the 1-way ANOVA Dunnett multiple comparison test and is related to the vehicle control. $* P<0.05 ; * * P<0.01$; $* * * P<0.001, \mathrm{P}<0.0001$. B MoDCs generated in the presence of Idelalisib or DMSO were partly stimulated with LPS on day 6. $24 \mathrm{~h}$ later, protein was isolated, and lysates were analyzed for Rel-B, c-Rel and Rel-A expression. Representative western blots show upregulation of
Rel-a, Rel-B and c-Rel by TLR stimulation. Rel-B and c-Rel expression is dose-dependently inhibited by Idealisib. Rel-A expression is unaffected by Idealisib exposure and serves as a loading control. C Protein was harvested from pretreated MoDCs as described above. Immunoprecipitates were analyzed for expression of PI3K, phosphorylated PI3K, phosphorylated STAT3 and $\beta$ Actin by western blotting. Representative blots are shown. Idelalisib does not affect expression of PI3K and p-STAT3, but phosphorylation of PI3K is inhibited by Idealisib. Equal protein amounts are confirmed by $\beta$ Actin expression 
complications are frequent [5]. Therefore, cytomegalovirus (CMV) monitoring as well as pneumocystis jirovecii pneumonia (PJP) prophylaxis are now mandatory. These infectious complications have led to a decreased and very cautious use of Idelalisib, and its replacement, by other new compounds in many cases in clinical routine [5]. However, the exact mechanisms mediating the immunosuppressive potential of Idelalisib have not been elucidated in detail yet.

Our report highlights that not only $\mathrm{T}$ cells and malignant B cells are targeted by PI3K $\delta$ inhibition but also DC differentiation and function are deeply modified by Idelalisib exposure in vitro. Differentiation of monocytes into moDCs using GM-CSF and IL-4 was markedly impaired by Idelalisib, which modulated proper DC differentiation and phenotype. For example, expression of CD83, which identifies mature DCs and is the most potent co-stimulatory molecule in the induction of allogeneic $\mathrm{T}$ cell proliferation, was impaired upon LPS exposure and Idelalisib treatment [10]. A decrease of CD83 expression due to Idelalisib co-culture may therefore emphasize the immature phenotype of these cells as well as their inability to induce $\mathrm{T}$ cell proliferation. As shown previously by a siRNA approach targeting human DCs in vitro [11], the downregulation of CD83 alone is sufficient for impaired $\mathrm{T}$ cell responses, even if expression of other co-stimulatory molecules is unaffected. The impaired DC function is further emphasized by diminished IL-12, IL-13 and TNF $\alpha$ secretion after LPS challenge. IL-13 production by DCs is important to maintain cytokine production in T helper 2 (Th2) cells [12]. IL-4, which is unchanged by Idelalisib treatment in mature moDCs, induces Th2 differentiation [13]. IL-13, in contrast, orchestrates survival and functionality of Th2 cells $[12,14]$. Th2-type cytokines are increased during CMV infection [15] and proper cytokine production by Th2 cells, which is maintained by IL-13 derived from DCs, is necessary for CMV clearance.

IL-12 is a central cytokine in the induction of $\mathrm{T}$ cell responses [16], and adequate release of IL-12 is required to control primary CMV infection in a CD4 + T cell-dependent manner [17]. Aside from lymphopenia, the impaired release of IL-12 and IL-13 by DCs may thus contribute to the increased susceptibility of Idelalisib-treated patients to CMV infection. Additionally, we were able to show that direct inhibition of the NFkB pathway may be one-way how Idelalisib impairs DC function and phenotype. In line with our data, previous studies could underline the importance of the NFKB pathway in DC development and IL-12 production [18].

Another serious complication during Idelalisib treatment is PJP infection. It has been shown previously that TNF $\alpha$ is required for clearance of pneumocystis jirovecii [19] and reduced TNF $\alpha$ levels, as shown here, may be why Idelalisib treatment increases the risk of fatal PJP infection. However, our results are limited because we only investigated the effect of Idelalisib on DC function and not on macrophages, which are a biologically significant source for $\mathrm{TNF} \alpha$.

Last, the inability of Idelalisib pretreated DCs to induce robust immune responses is stressed by the fact that DCs exposed to Idelalisib improperly stimulate allogeneic $\mathrm{T}$ cell proliferation, which is another prerequisite to induce a robust immune response after an infectious challenge. DCs activate naïve $T$ cells via 3 signals: Antigen presentation (signal 1), co-stimulation (signal 2) and cytokine production (signal 3) [20]. Although we did not investigate antigen presentation, we could unveil modulation of co-stimulation and cytokine production by Idelalisib, which is further supported by our results regarding the induction of allogeneic $\mathrm{T}$ cell proliferation.

Our results may help to understand the restricted use of Idelalisib due to fatal infectious complications. Idelalisib modulates the expression of at least one co-stimulatory molecule and, more importantly, diminishes cytokine production by DCs in vitro. These cytokines are essential for proper $\mathrm{T}$ cell differentiation and polarization. Clinical infection with CMV and PCP may therefore not only be caused by defective $\mathrm{T}$ cell responses, but also by insufficient $\mathrm{T}$ cell polarization due to diminished cytokine release. Although our data were generated in vitro, clinical observations implicate relevance for our findings in vivo.

In contrast, suppression of T cells and DCs may open new therapeutic venues for PI3K $\delta$ inhibition. For example, in myeloproliferative neoplasms, which are driven by chronic inflammation [21], a clinical trial combining PI3K $\delta$ inhibition and standard treatment with the JAK inhibitor Ruxolitinib showed promising interim results in patients with myelofibrosis (ClinicalTrials.gov Identifier: NCT02718300). These promising results are supposed to be caused by direct effects of PI3K inhibition on malignant cells [22], but additional effects as shown in this work have to be investigated as well.

Authors' contribution CB and SND performed experiments, SS and $\mathrm{AH}$ analyzed results, made the figures and wrote the manuscript, $\mathrm{AH}$ and $\mathrm{PB}$ designed the research and discussed results.

Funding Open Access funding enabled and organized by Projekt DEAL. This work was funded by the Deutsche Krebshilfe through a Mildred Scheel Nachwuchszentrum Grant (Grant number 70113307 to S.S.) and supported by a grant from the Deutsche Forschungsgemeinschaft (DFG EXC2151-390873048 to A.H.).

Data availability The datasets generated during and analyzed during the current study are available from the corresponding author on reasonable request. 


\section{Declarations}

Conflict of interest The authors declare that they have no conflict of interests.

Ethics approval The study was conducted in accordance with the Declaration of HELSINKI and approved by the institutional ethics committee of the University of Bonn. (\#173/09).

Open Access This article is licensed under a Creative Commons Attribution 4.0 International License, which permits use, sharing, adaptation, distribution and reproduction in any medium or format, as long as you give appropriate credit to the original author(s) and the source, provide a link to the Creative Commons licence, and indicate if changes were made. The images or other third party material in this article are included in the article's Creative Commons licence, unless indicated otherwise in a credit line to the material. If material is not included in the article's Creative Commons licence and your intended use is not permitted by statutory regulation or exceeds the permitted use, you will need to obtain permission directly from the copyright holder. To view a copy of this licence, visit http://creativecommons.org/licenses/by/4.0/.

\section{References}

1. Armitage JO, Gascoyne RD, Lunning MA, Cavalli F (2017) Nonhodgkin lymphoma. The Lancet 390:298-310. https://doi.org/10. 1016/S0140-6736(16)32407-2

2. Herman SEM, Gordon AL, Wagner AJ et al (2010) Phosphatidylinositol 3-kinase- $\delta$ inhibitor CAL-101 shows promising preclinical activity in chronic lymphocytic leukemia by antagonizing intrinsic and extrinsic cellular survival signals. Blood 116:20782088. https://doi.org/10.1182/blood-2010-02-271171

3. Furman RR, Sharman JP, Coutre SE et al (2014) Idelalisib and rituximab in relapsed chronic lymphocytic leukemia. N Engl J Med 370:997-1007. https://doi.org/10.1056/NEJMoa1315226

4. Gopal AK, Kahl BS, de Vos S et al (2014) PI3Kס inhibition by idelalisib in patients with relapsed indolent lymphoma. N Engl J Med 370:1008-1018. https://doi.org/10.1056/NEJMoa1314583

5. de Weerdt I, Koopmans SM, Kater AP, van Gelder M (2017) Incidence and management of toxicity associated with ibrutinib and idelalisib: a practical approach. Haematologica 102:1629-1639. https://doi.org/10.3324/haematol.2017.164103

6. Martinelli S, Maffei R, Fiorcari S et al (2018) Idelalisib impairs T-cell-mediated immunity in chronic lymphocytic leukemia. Haematologica 103:e598-e601. https://doi.org/10.3324/haema tol.2017.187070

7. Carbone FR, Kurts C, Bennett SRM et al (1998) Cross-presentation: a general mechanism for CTL immunity and tolerance. Immunol Today 19:368-373. https://doi.org/10.1016/S01675699(98)01301-2

8. Heine A, Held SAE, Daecke SN et al (2013) The JAKinhibitor ruxolitinib impairs dendritic cell function in vitro and in vivo. Blood 122:1192-1202. https://doi.org/10.1182/ blood-2013-03-484642

9. Koerber R-M, Held SAE, Heine A et al (2015) Analysis of the anti-proliferative and the pro-apoptotic efficacy of Syk inhibition in multiple myeloma. Exp Hematol Oncol. https://doi.org/10. 1186/s40164-015-0016-z

10. Zhou LJ, Tedder TF (1995) Human blood dendritic cells selectively express CD83, a member of the immunoglobulin superfamily. J Immunol 154:3821-3835

11. Aerts-Toegaert C, Heirman C, Tuyaerts S et al (2007) CD83 expression on dendritic cells and T cells: correlation with effective immune responses. Eur J Immunol 37:686-695. https://doi. org/10.1002/eji.200636535

12. Bellinghausen I, Brand P, Böttcher I et al (2003) Production of interleukin-13 by human dendritic cells after stimulation with protein allergens is a key factor for induction of T helper 2 cytokines and is associated with activation of signal transducer and activator of transcription-6. Immunology 108:167-176. https://doi.org/10. 1046/j.1365-2567.2003.01576.x

13. Junttila IS (2018) Tuning the cytokine responses: an update on Interleukin (IL)-4 and IL-13 receptor complexes. Front Immunol. https://doi.org/10.3389/fimmu.2018.00888

14. Yang L, Xu L-Z, Liu Z-Q et al (2016) Interleukin-13 interferes with activation-induced t-cell apoptosis by repressing p53 expression. Cell Mol Immunol 13:669-677. https://doi.org/10.1038/cmi. 2015.50

15. Essa S, Pacsa A, Raghupathy R et al (2009) Low levels of Th1type cytokines and increased levels of Th2-type cytokines in kidney transplant recipients with active cytomegalovirus infection. Transplant Proc 41:1643-1647. https://doi.org/10.1016/j.trans proceed.2008.10.098

16. Trinchieri G (2003) Interleukin-12 and the regulation of innate resistance and adaptive immunity. Nat Rev Immunol 3:133-146. https://doi.org/10.1038/nri1001

17. Popescu I, Pipeling MR, Mannem H et al (1950) (2016) interleukin 12-dependent cytomegalovirus-specific CD4+ T cell proliferation, T-bet induction and effector multi-function during primary infection are key determinants for early immune control. J Immunol Baltim Md 196:877-890. https://doi.org/10.4049/jimmunol. 1501589

18. Ouaaz F, Arron J, Zheng Y et al (2002) Dendritic cell development and survival require distinct NF- $\mathrm{\kappa B}$ subunits. Immunity 16:257-270. https://doi.org/10.1016/S1074-7613(02)00272-8

19. Chen W, Havell EA, Harmsen AG (1992) Importance of endogenous tumor necrosis factor alpha and gamma interferon in host resistance against Pneumocystis carinii infection. Infect Immun 60:1279-1284

20. Granucci F, Lutz MB, Zanoni I (2013) The nature of activatory and tolerogenic dendritic cell-derived signal 2. Front Immunol. https://doi.org/10.3389/fimmu.2013.00198

21. Hasselbalch HC, Bjørn ME (2015) MPNs as inflammatory diseases: the evidence, consequences, and perspectives. Mediators Inflamm. https://doi.org/10.1155/2015/102476

22. Bartalucci N, Calabresi L, Balliu M et al (2017) Inhibitors of the $\mathrm{PI} 3 \mathrm{~K} / \mathrm{mTOR}$ pathway prevent STAT5 phosphorylation in JAK2V617F mutated cells through PP2A/CIP2A axis. Oncotarget 8:96710-96724

Publisher's Note Springer Nature remains neutral with regard to jurisdictional claims in published maps and institutional affiliations. 